\title{
RECENT DEVELOPMENTS IN MM AND SUB-MM
}

\section{INTERFEROMETRY}

\author{
M. ISHIGURO \\ Nobeyama Radio Observatory, Nobeyama, Minamisaku, Nagano 384-13, Japan
}

\begin{abstract}
Four existing millimeter arrays have been very productive in the last few years and are currently being expanded in their capabilities. Recent technical developments in these arrays as well as the prospects for submillimeter interferometry and larger arrays are presented.
\end{abstract}

Key words: Interferometry - Millimeter Wave - Submillimter Wave

\section{Introduction}

Millimeter wave interferometry has been demonstrated as a major tool for studying fine structures of molecular line emission and continuum emission in various astronomical objects. There are four operating millimeter arrays in the world; BIMA(Berkeley-Illinois-Maryland-Association) Array, Owens Valley Millimeter Array, NMA(Nobeyama Millimeter Array), and IRAM Array. To review recent technical developments and new astronomical results with these arrays, IAU Colloquium 140, Astronomy with Millimeter and Submillimeter Wave Interferometry, was held in October,1992 at Hakone, Japan . It was evident from the many papers presented at this conference that these operating arrays have been very productive in the past two or three years and are currently being expanded in their capabilities. In the submillimeter wavelengths, the first interfeometric observation was conducted with JCMT 15-m and CSO 10-m telescopes at $321 \mathrm{GHz}$, while the Smithsonian submillimeter array will start its construction at Mauna Kea. Recent technical developments in millimeter and submillimeter interferometry are reviewed mostly from the papers presented at the Hakone conference.

\section{Expansions of the existing arrays}

The existing arrays are currently being expanded in their capabilities by adding more antennas and high frequency receivers and by extending the bandwidth of digital correlators. The expansion will complete within one or two years. Table 1 briefly describes the performances of the expanded millimeter-wave arrays. Two extremes are the IRAM array which has the largest collecting area equivalent to a 30-m single dish and the BIMA array which is the largest in simultaneously available baselines (36).

\subsection{BIMA ARRAY}

The BIMA array is currently being expanded from three to nine antennas(Welch 1993). With 9-element array, transportation of antennas is not necessary for most of the observations. The new 6-m antennas are expected to have a surface accuracy better than $20 \mu \mathrm{m}$ rms and employ roller drives with no backlash. In addition, the IF bandwidth has increased to $830 \mathrm{MHz}$, operation at $210-270 \mathrm{GHz}$ with SIS receivers is being added, the spectral line correlator maximum bandwidth is now 
TABLE I

Properties of the expanded millimeter arrays.

\begin{tabular}{lllll}
\hline & BIMA & OVRO & NMA & IRAM \\
\hline & & & & \\
Location & Hat Creek & Owens Valley & Nobeyama & Plateau de Bure \\
Altitude & $1024 \mathrm{~m}$ & $1200 \mathrm{~m}$ & $1350 \mathrm{~m}$ & $2553 \mathrm{~m}$ \\
Baselines & $300 \mathrm{~m} \mathrm{EW}$ & $200 \mathrm{~m} \mathrm{EW}$ & $560 \mathrm{~m} \mathrm{EW}$ & $288 \mathrm{~m} \mathrm{EW}$ \\
& $200 \mathrm{~m} \mathrm{NS}$ & $220 \mathrm{~m} \mathrm{NS}$ & $520 \mathrm{~m} \mathrm{NS}$ & $160 \mathrm{~m} \mathrm{NS}$ \\
Antenna & $6 \mathrm{~m} \mathrm{x}$ & $10 \mathrm{~m} \mathrm{x} 6$ & $10 \mathrm{~m} \mathrm{x}$ & $15 \mathrm{~m} \mathrm{x}$ \\
Surface Accuracy & $20 \mu \mathrm{m}$ & $35 \mu \mathrm{m}$ & $40-70 \mu \mathrm{m}$ & $70 \mu \mathrm{m}$ \\
Receiver & SIS & SIS & SIS & SIS \\
Wavelengths & $2.6 \mathrm{~mm}$ & $2.6 \mathrm{~mm}$ & $2.6 \mathrm{~mm}$ & $2.6 \mathrm{~mm}$ \\
& $1.3 \mathrm{~mm}$ & $1.3 \mathrm{~mm}$ & $2.0 \mathrm{~mm}$ & $1.3 \mathrm{~mm}$ \\
& & & $1.3 \mathrm{~mm}$ & \\
Bandwidth & $800 \mathrm{MHz}$ & $512 \mathrm{MHz}$ & $2000(320) \mathrm{MHz}$ & $960 \mathrm{MHz}$ \\
No. of Channels & 512 & 256 & $256(1024)$ & 3072 \\
\hline
\end{tabular}

$800 \mathrm{MHz}$. The new version of the correlator is based on the NFRA correlator chip (Bos 1987). The four window bandwidths may now be set to $200 \mathrm{MHz}, 100 \mathrm{MHz}$, $50 \mathrm{MHz}, 25 \mathrm{MHz}, 12.5 \mathrm{MHz}$, or $6.25 \mathrm{MHz}$, with the use of time multiplexing to achieve the wider bandwidths. Each window may be set to any frequency in the $830 \mathrm{MHz}$ IF band.

\subsection{OVRO ARRAY}

The Owens Valley Millimeter Array is an aperture synthesis array consisting of 10.4 $\mathrm{m}$ diameter telescope(Scoville 1993). Originally bult as a three element array, it is currently undergoing a major expansion to six telescopes. The $10.4 \mathrm{~m}$ telescopes have space-frame backstructures and aluminum honey-comb panels. Each telescope is equipped with SIS receivers for both $80-115 \mathrm{GHz}$ and $215-270 \mathrm{GHz}$ bands. New optics have been installed to permit rapid switching between the bands in order to exploit the best weather conditions and enable phase calibration at $\lambda \sim 3 \mathrm{~mm}$ during $\lambda \sim 1 \mathrm{~mm}$ observations. The system temperatures in single sideband are $\sim$ $400 \mathrm{~K}$ at $115 \mathrm{GHz}$ and $\sim 600 \mathrm{~K}$ at $230 \mathrm{GHz}$. For the tracking delay, a wideband $(0.5-3.5 \mathrm{GHz})$ system has recently been developed. For the three longest segments the delays are optical fiber; the remaining shorter delay segments are coaxial and microstrip transmission lines ( Padin and Soares 1993).

\subsection{NMA}

A new 10-m antenna is under construction at Nobeyama to expand NMA to 6element. The surface accuracy of the new antenna is expected to be $\leq 40 \mu \mathrm{m}$ rms. The surface panel is an aluminum honeycome type and $20 \mu \mathrm{m}$ rms has been achieved. Each panel is supported by six mortorized actuators and can be remotely adjusted. The gravitational deformation in the backup structure is estimated to 
be $\leq 10 \mu \mathrm{m}$ rms. The optics is designed to be efficient at $100-200 \mathrm{GHz}$. The illumination of the telescope aperture is controlled in the beam-waveguide system to obtain higher aperture efficiency in this frequency band. Tunerless SIS mixers at $100 \mathrm{GHz}$ and $150 \mathrm{GHz}$ have been used successfully. The same type of SIS mixers at $230 \mathrm{GHz}$ are being developed at Nobeyama. The FX correlators has $320 \mathrm{MHz}$ maximum bandwidth with 1024 channels, and has been used for both spectralline and continuum observations( Chikada et al. 1987). To extend the maximum bandwidth to $2 \mathrm{GHz}$, new XF type correlator chips are being developed to be used with a $4 \mathrm{Gs} / \mathrm{sec}$ A/D converter (Morita 1993).

\subsection{IRAM ARRAY}

The fourth 15-m antenna is under construction at Plateau de Bure. The telescope design is the same as the existing antennas. This antenna adopted curbon fiber technology for the surface panels and the main-refrector structure to reduce the thermal deformations. The large collecting area of 15-m antennas has an advantage in calibrating visibility phase and makes the IRAM array very unique in mapping very weak sources. The field of view, however, is limited to 40 arcsecond, so that mapping of extended molecular clouds must be done by mosaicing. The minimum detectable flux density is $2 \mathrm{mJy}$ with one hour integration at $90 \mathrm{GHz}$. Recently, new correlator system has been completed. The correlator consists of six idential units; each unit has $160 \mathrm{MHz}$ maximum bandwidths and very flexible operation is possible. The number of channels are 64 and 256 for $160 \mathrm{MHz}$ bandwidth and 20 $\mathrm{MHz}$ bandwidth, respectively(Guilloteau 1993).

\section{New technical developments}

In this section, some new technical developments are described which are not fully included in the previous section.

\subsection{SUBMILLIMETER INTERFEROMETRY}

A linked submillimeter-wave interferometer using the CSO $10.4 \mathrm{~m}$ and the JCMT $15 \mathrm{~m}$ telescopes located on Mauna Kea at an elevation of $4200 \mathrm{~m}$, has been built and tested successfully (Carlstrom et al. 1992). The large collecting area, the low atmospheric opacity at Mauna Kea, and the high quality of the receivers available at both observatories, leads to impressive sensitivity. The nealy east-west $165 \mathrm{~m}$ baseline sets a resolution limit of 0.6 " at $870 \mu \mathrm{m}$. Optical fibers are used to link the two telescopes and their amplitude and phase stability are excellent.

The Smithsonian Astrophysical Observatory plans to construct a submillimeter interferometer array(SMA) at Mauna Kea to operate from $\sim \lambda 1.3 \mathrm{~mm}$ to $\sim \lambda 0.3$ $\mathrm{mm}$ (Ho 1993). With this instrument, ground-based radio astronomy will have complete spectral coverage at better than one arcsecond resolution up to the atmospheric limit of $\sim \lambda 0.3 \mathrm{~mm}$. SMA consists of six movable 6-m antennas, with a baseline up to $500 \mathrm{~m}$. A typical angular resolution will be $0.1-0.5$ arcsecond at $\lambda 0.35 \mathrm{~mm}$. Carbon fiber technology will be used extensively to achive highly efficient antennas. 


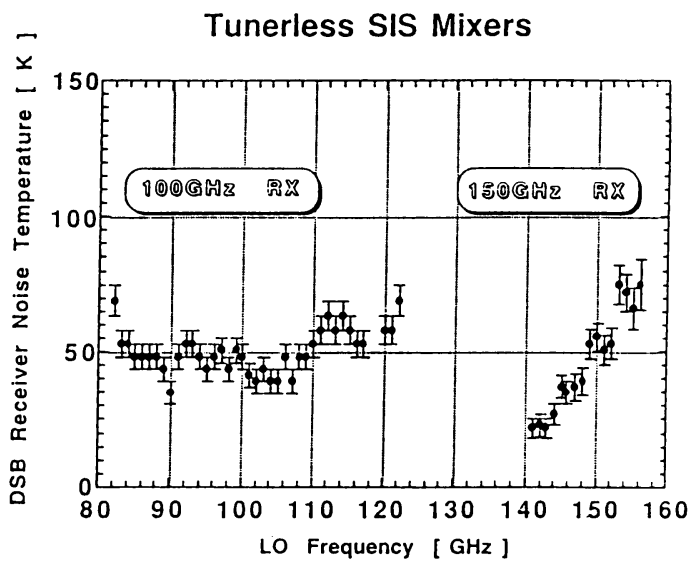

Fig. 1. Noise performance of the tunerless SIS mixers(Sunada K. 1993).

\subsection{SIS RECEIVER}

SIS receivers have been extensively used for millimeter arrays to achieve low receiver temperatures(Phillips, T. 1993). The Nb SIS junction has proved its high reliability. The mixer noise temperature are now $\sim 10 \mathrm{~h} \nu / \mathrm{k}(\mathrm{h} \nu / \mathrm{k}$ : quantum limit) and will be improved to $\sim 3 \mathrm{~h} \nu / \mathrm{k}$ in the future. Very low noise temperatures have been realized even with tunerless SIS mixers. Figure 1 shows the noise performance of the 100 $\mathrm{GHz}$ and $150 \mathrm{GHz}$ tunerless SIS mixers at Nobeyama( Sunada K. 1993). A new type of Gifford-Mcmahon refrigerator is developed at Toshiba, using a new regenerator material $\mathrm{Er} 3 \mathrm{Ni}$, and the lowest temperature achieved is $2.5 \mathrm{~K}$. This type of G-M refregerator is being used for the Nobeyama 45-m telescope(Kuriyama et al.1993) and the BIMA array(Welch 1993).

\subsection{Signal Transmission and Processing}

Coaxial cables have been used for the transmission of the reference signal to phaselock the local oscillators and IF return signal. Specially designed phase-stabilized coaxial cables have temperature coefficient of $\sim 1 \mathrm{ppm} /{ }^{\circ} \mathrm{C}$.

Optical fiber links are attractive for IF transmission because of wide bandwidths and small passband errors. Phase-stabilized optical fiber cable is realized by a liquid crystal polymer coating which has a thermal expansion coefficient that compensates for that of the silica fiber ( Tanaka et al. 1992 ). The delay variation of this fiber cable with temperature is $\sim 0.04 \mathrm{ppm} /{ }^{\circ} \mathrm{C}$, which is 100 times smaller than that of coaxial cables( see Figure 2). Phase drift in the $\mathrm{E} / \mathrm{O}$ and $\mathrm{O} / \mathrm{E}$ converters are also very small and comparable to that in an 1-km fiber cable(see Figure 3).

In the Owens Valley Millimeter Array, a wideband (0.5 - 3.5 GHz) delay tracking system has recently been developed. For the three longest segments (512, 256 and $128 \mathrm{~ns}$ ) the delays are realized in optical fiber; the remaining shorter delay segments are coaxial and microstrip transmission lines (Soares and Padin 1993). The fiber 


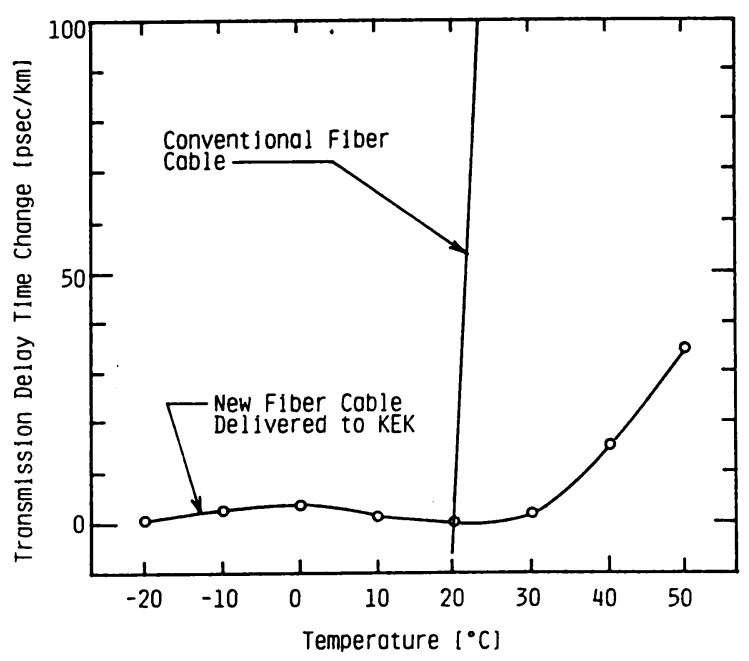

Fig. 2. Delay variation of the phase-stabilized optical fiber(Tanaka et al. 1992).

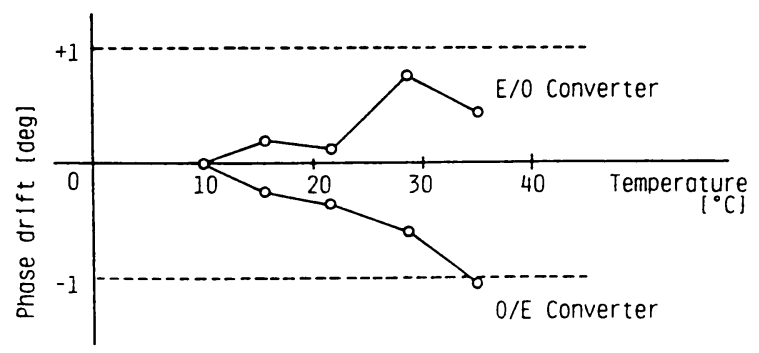

Fig. 3. Phase drift at $600 \mathrm{MHz}$ in the $\mathrm{E} / \mathrm{O}$ and $\mathrm{O} / \mathrm{E}$ converters(Tanaka et al. 1992).

optic link is also used for the JCMT-CSO Interferometer( Lay, Hills, and Carlstrom 1993).

\subsection{Atmospheric Problems and Calibrations}

The principal limitation in achieving high angular resolution is the atmospheric phase fluctuation. Fluctuations at higher frequencies are caused by water vapor in the atmosphere. Recent measurements at the Plateau de Bure shows that a mean resolution limit of about two arc seconds at $1 \mathrm{~mm}$ (Olmi and Downes 1992). Some novel phase calibration schemes are considered for a large array: determining the atmospheric phase from total power fluctuations, frequent beam switching onto a nearby calibrator, and spatially pairing the antennas such that one antenna is observing the calibrator while the other antenna is observing the target source(Holdaway 1993). In the first method, the principal technical challenge is the stabilization of the system gain to the $10^{-4}$ level over time interval of $15-30$ min- 


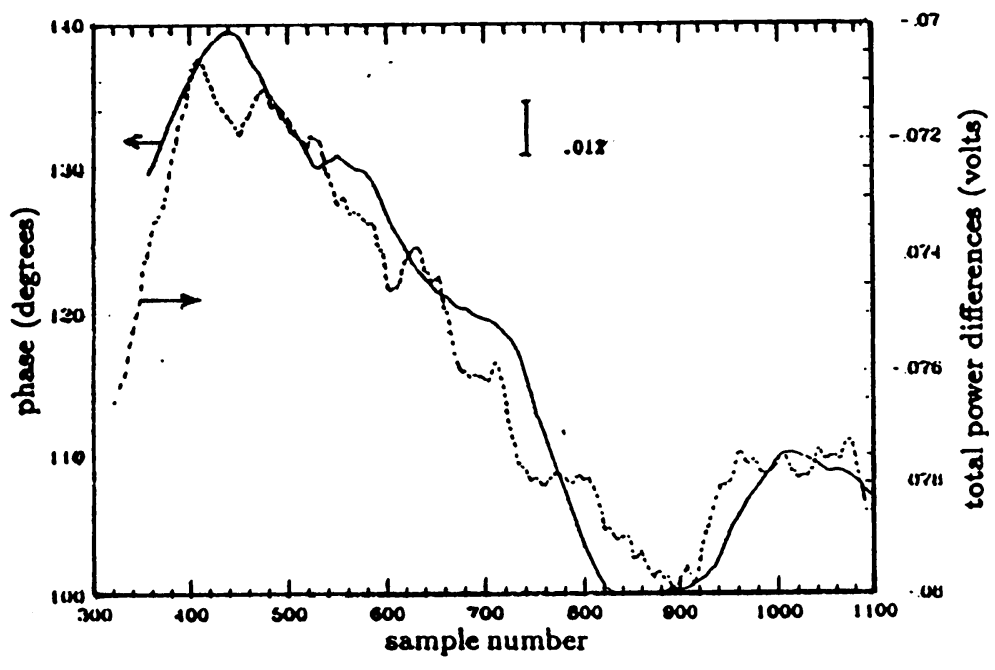

Fig. 4. An example of the correlation between antenna total power and visibility phase(Welch 1993).

utes(Welch 1993). Figure 4 shows an example of the correlation between antenna total power and visibility phase.

\section{Future plans for large array}

There are three major plans for large millimeter and submillimeter arrays; MMA (Millimeter Array) proposed by NRAO, USA(Brown 1993), LMA (Large Millimeter Array) proposed by NRO, Japan(Ishiguro 1993), and SHMA (Southern Hemisphere Millimeter Array) proposed by European countries(Booth 1993). Table 2 describes the performance parameters for the proposed arrays.

TABLE II

Future plans for large millimeter and submillimeter arrays.

\begin{tabular}{llll}
\hline & MMA & LMA & SHMA \\
\hline & & & \\
Antenna & $8 \mathrm{~m} \mathrm{x} \mathrm{40}$ & $10 \mathrm{~m} \times 50$ & $15 \mathrm{~m} \times 40(10 \mathrm{~m} \mathrm{x} \mathrm{90)}$ \\
Collecting Area & $2000 \mathrm{~m}^{2}$ & $4000 \mathrm{~m}^{2}$ & $7000 \mathrm{~m}^{2}$ \\
Frequency Range & $35-350 \mathrm{GHz}$ & $40-500 \mathrm{GHz}$ & $40-350 \mathrm{GHz}$ \\
Maximum Baseline & $3 \mathrm{~km}$ & $2-3 \mathrm{~km}$ & $10 \mathrm{~km}$ \\
\hline
\end{tabular}

To achieve $0.1-0.5$ arcseond resolutions in these arrays, the site selection is essential. So far, site surveys have been based on the opacity measurements in which atmospheric phase stability has been evaluated indirectly. Recently, two groups, 


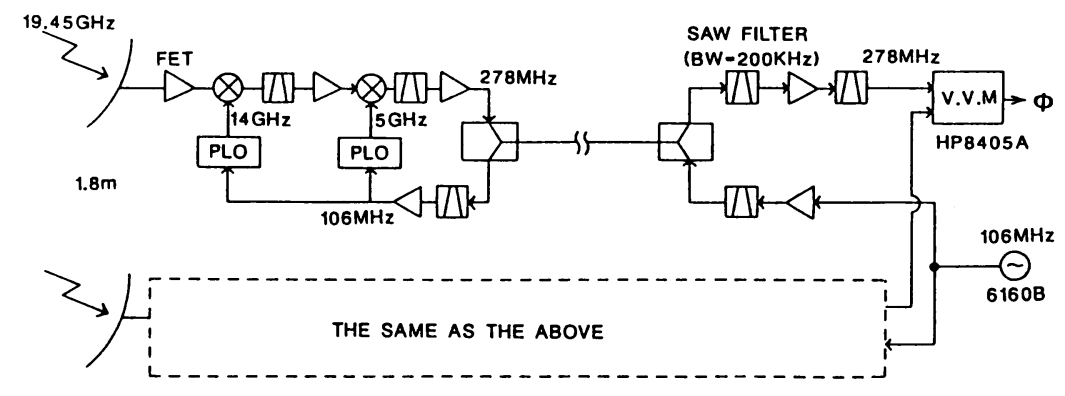

Fig. 5. Radio seeing monitor at Nobeyama.

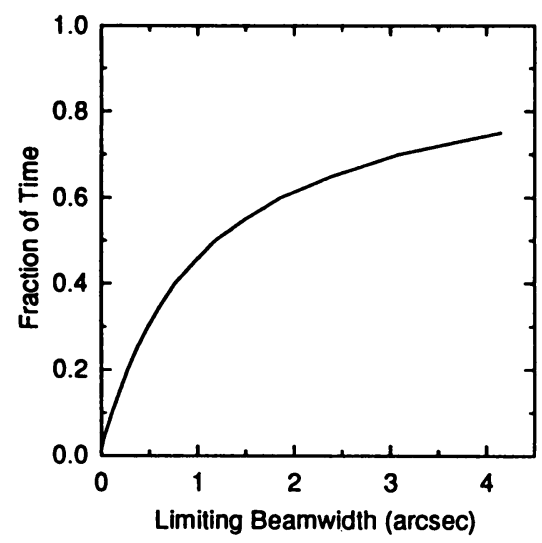

Fig. 6. The limiting resolution of an interferometer on Mauna Kea at $345 \mathrm{GHz}$. The values are extrapolated from measurements at $100 \mathrm{~m}$ baseline(Masson 1993).

one at NRO (Ishiguro et al. 1990), and one at SAO (Masson 1993) have used small interferometers (Radio seeing monitor) observing geostationary satellite signals to make continuous measurements of atmospheric phase fluctuations. Figure 5 is an example for the radio seeing monitor at Nobeyama (Ishiguro et al. 1990). Figure 6 shows the limiting resolution of an interferometer on Mauna Kea at $345 \mathrm{GHz}$ estimated from the monitoring of radio seeing (Masson 1993). A more compact radio seeing monitor is under development at Nobeyama to be used for the site surveys for LMA.

\section{References}

Booth, R. S. : 1993, in Astronomy with Millimeter and Submilimeter Wave Interferometry, eds. M. Ishiguro and Wm J. Welch, Astron. Soc. of the Pacific Conference Series, Sanfrancisco, in press.

Bos, A. : 1987, NFRA ITR no.178.

Brown, R. : 1993, in Astronomy with Millimeter and Submilimeter Wave Interferometry, eds. M. Ishiguro and Wm J. Welch, Astron. Soc. of the Pacific Conference Series, Sanfrancisco, in press. 
Carlstrom, J. E. et al. : 1993, in Astronomy with Millimeter and Submilimeter Wave Interferometry, eds. M. Ishiguro and Wm J. Welch, Astron. Soc. of the Pacific Conference Series, Sanfrancisco, in press.

Chikada et al.: 1987, Proc. IEEE 75, 1203

Guilloteau, S. : 1993, in Astronomy with Millimeter and Submilimeter Wave Interferometry, eds. M. Ishiguro and Wm J. Welch, Astron. Soc. of the Pacific Conference Series, Sanfrancisco, in press.

Hills, R. E. : 1993, in Astronomy with Millimeter and Submilimeter Wave Interferometry, eds. M. Ishiguro and Wm J. Welch, Astron. Soc. of the Pacific Conference Series, Sanfrancisco, in press.

Ho, P. T. P. : 1993, in Astronomy with Millimeter and Submilimeter Wave Interferometry, eds. M. Ishiguro and Wm J. Welch, Astron. Soc. of the Pacific Conference Series, Sanfrancisco, in press.

Holdaway, M. A. : 1993, in Astronomy with Millimeter and Submilimeter Wave Interferometry, eds. M. Ishiguro and Wm J. Welch, Astron. Soc. of the Pacific Conference Series, Sanfrancisco, in press.

Ishiguro, M. : 1993, in Astronomy with Millimeter and Submilimeter Wave Interferometry, eds. M. Ishiguro and Wm J. Welch, Astron. Soc. of the Pacific Conference Series, Sanfrancisco, in press.

Ishiguro, M., Kanzawa, T.,and Kasuga, T. : 1990, in URSI/IAU Symp. on Radio Astronomical Seeing, eds. J. E. Baldwin and Wang Shouguan, IAP, Beijing, 60.

Kuriyama, T., Hatakeyama, H., Takahashi, M., Nakagome, H., Kawabe, R., Iwashita, H., McCulloch, G., Shibata, K., and Ukita, N. : 1993, in Astronomy with Millimeter and Submilimeter Wave Interferometry, eds. M. Ishiguro and Wm J. Welch, Astron. Soc. of the Pacific Conference Series, Sanfrancisco, in press.

Lay, O. P., Hills, R. E., and Carlstrom, J. E. : 1993, in Astronomy with Millimeter and Submilimeter Wave Interferometry, eds. M. Ishiguro and Wm J. Welch, Astron. Soc. of the Pacific Conference Series, Sanfrancisco, in press.

Masson, C. R. : 1993, in Astronomy with Millimeter and Submilimeter Wave Interferometry, eds. M. Ishiguro and Wm J. Welch, Astron. Soc. of the Pacific Conference Series, Sanfrancisco, in press.

Morita, K.-I. : 1993, in Astronomy with Millimeter and Submilimeter Wave Interferometry, eds. M. Ishiguro and Wm J. Welch, Astron. Soc. of the Pacific Conference Series, Sanfrancisco, in press.

Olmi, L., and Downes, D.: 1992, Astron. Astrophys., in press. ,

Padin, S. and Soares, S. F. : 1993, in Astronomy with Millimeter and Submilimeter Wave Interferometry, eds. M. Ishiguro and Wm J. Welch, Astron. Soc. of the Pacific Conference Series, Sanfrancisco, in press.

Tanaka S., Murakami, Y., Sato, Y., and Urakawa, J.: 1990, KEK Report, National Laboratory for High Energy Physics, Japan 90-5, 385

Soares, S. F. and Padin, S. : 1993, in Astronomy with Millimeter and Submilimeter Wave Interferometry, eds. M. Ishiguro and Wm J. Welch, Astron. Soc. of the Pacific Conference Series, Sanfrancisco, in press.

Sunada K. : 1993, in Astronomy with Millimeter and Submilimeter Wave Interferometry, eds. M. Ishiguro and Wm J. Welch, Astron. Soc. of the Pacific Conference Series, Sanfrancisco, in press.

Welch, Wm. J. : 1993, in Astronomy with Millimeter and Submilimeter Wave Interferometry, eds. M. Ishiguro and Wm J. Welch, Astron. Soc. of the Pacific Conference Series, Sanfrancisco, in press.

Napier:

Discussion:

For the upgraded Nobeyama millimeter interferometer antennas, how does the shaping of the beam waveguide mirrors work, and what improvement in antenna efficiency is expected?

Ishiguro:

The shaping will be done with mirrors in the beam waveguide system, not in the main and sub-reflectors. The expected aperture efficiency is $\sim 80-90 \%$. 